\title{
Dynamic Time Scales in Colored Glass Nuclear Matter
}

\author{
V. Parihar, A. Widom, Y. N. Srivastava $\dagger$ \\ Physics Department, Northeastern University, Boston MA USA \\ and \\ $†$ Physics Department \& INFN, University of Perugia, Perugia IT
}

\section{Introduction}

Ultra high energy collisions between heavy nuclei can be employed to experimentally probe the QCD properties of dense quark-gluon systems [1, 2, 3, 4, 5]. The properties of the high density quark gluon system have also been compared to a color glass[6, 7, 8, 9, 10, 11, 12, 13]. High energy proton-proton scattering also probes the $\mathrm{QCD}$ vacuum. The QCD vacuum differs from the $\mathrm{QED}$ vacuum via properties largely due to the nature of the electrically charged and color charged dielectric screening response functions. In the QCD case, there is a quark-quark potential containing both a Coulomb-like piece as well as a linear confining potential. The confining force may be viewed in terms of the tension $\sigma$ in a "string" connecting two quarks having the experimental value

$$
\sigma \approx \frac{0.18 \mathrm{GeV}^{2}}{\hbar c} \approx 1.45 \times 10^{5} \text { Newton }
$$

The QCD string may be visualized as a color electric field flux tube. The string vibrational and rotational states describe the mesons contributing to nuclear forces. The QCD string has a much lower tension than some theoretical strings said to describe gravity; e.g.

$$
\sigma_{\text {gravity }}=\frac{c^{4}}{8 \pi G} \approx 4.8 \times 10^{42} \text { Newton. }
$$

In what follows only properties of the low tension QCD string will be invoked. 
As the string excitation energies grow ever larger, the enormous number of possible string excitations can be described by the entropy as a function of energy. In computing quantum transitions, the number $\Omega$ of possible quantum states is determined by the entropy $S$ via

$$
S=k_{B} \ln \Omega .
$$

Since one must average over initial states and sum over the final states, transition rates have the "detailed balance" form

$$
\Gamma_{i \rightarrow f}=v_{\infty}\left\{\frac{\Omega_{f}}{\Omega_{i}}\right\}=v_{\infty} \exp \left[\frac{S_{f}-S_{i}}{k_{B}}\right] .
$$

Eq.(4) will be applied to the time scales of nuclear matter motions which occur shortly after an ultra high energy collision between two heavy nuclei. The time scales are familiar from the the theory of glasses[14, 15, 16, 17]. The time scales $\operatorname{are}[18]$

$$
\tau=\tilde{\tau} \exp \left[\frac{\Phi}{k_{B}\left(T-T_{H}\right)}\right], \text { for } T>T_{H},
$$

wherein $T_{H}$ is the Hagedorn temperature[19] and $\Phi$ is the activation energy. Experimentally,

$$
k_{B} T_{H} \approx 207 \mathrm{MeV} \text { and } \Phi \equiv \frac{7 k_{B} T_{H}}{2} \approx 725 \mathrm{MeV} .
$$

The notion of final state particle product entropy is also of use in discussing the total cross section as a function of center of mass energy.

\section{Vacuum Stability and Dielectric Screening}

The Vacuum law of force between two charges $z_{1} e$ and $z_{2} e$ may be written as

$$
V(r)=e^{2} z_{1} z_{2} \int\left[\frac{e^{i \mathbf{Q} \cdot \mathbf{r}}}{Q^{2} \varepsilon\left(Q^{2}\right)}\right] \frac{d^{3} \mathbf{Q}}{(2 \pi)^{3}}=\left[\frac{z_{1} z_{2} e^{2}}{4 \pi r}\right] \chi(r),
$$

wherein the vacuum screening function $\chi(r)$ is determined by the vacuum dielectric response function $\varepsilon\left(Q^{2}\right)$ via

$$
\chi(r)=\frac{2}{\pi} \int_{0}^{\infty} \sin (Q r)\left[\frac{d Q}{Q \varepsilon\left(Q^{2}\right)}\right] .
$$


In the more usual notation of QED, one defines the dielectric response of the vacuum in terms of a running coupling strength

$$
\alpha=\frac{e^{2}}{4 \pi \hbar c} \Rightarrow \alpha\left(Q^{2}\right)=\frac{e^{2}}{4 \pi \hbar c \varepsilon\left(Q^{2}\right)} .
$$

In the limit of large distances, $\hbar / m c \ll r$, we have

$$
\lim _{Q^{2} \rightarrow 0^{+}} \varepsilon\left(Q^{2}\right)=\lim _{r \rightarrow \infty} \chi(r)=1,
$$

which implies a simple Coulomb law. The subtracted dispersion relation then reads

$$
\varepsilon\left(Q^{2}\right)=1-\frac{Q^{2}}{\pi} \int_{0}^{\infty}\left[\frac{\mathfrak{I} m \varepsilon\left(-v-i 0^{+}\right)}{\nu+Q^{2}}\right] \frac{d v}{v} .
$$

One has a dissipative QED vacuum with positive electrical conductivity for timelike wave vectors,

$$
\Im m \varepsilon\left(-v-i 0^{+}\right) \geq 0,
$$

at the expense of a Landau $\alpha\left(Q^{2}\right)$ singularity for some space-like wave vector implicit in Eqs.(9) and (12). Such a singularity has been referred to as a Landau ghost [20, 21, 22, 23].

For the QCD case the potential between color charges analogous to Eq.(7) may be written as

$$
\begin{array}{r}
V_{s}(r)=\eta^{a b} t_{1 a} t_{2 b}\left(\frac{g^{2}}{4 \pi r}\right) \chi_{s}(r), \\
\chi_{s}(r)=\frac{2}{\pi} \int_{0}^{\infty} \sin (Q r)\left[\frac{d Q}{Q \varepsilon_{s}\left(Q^{2}\right)}\right],
\end{array}
$$

wherein the strong screening dielectric response arises from the strong running coupling constant

$$
\alpha_{s}=\frac{g^{2}}{4 \pi \hbar c} \Rightarrow \alpha_{s}\left(Q^{2}\right)=\frac{g^{2}}{4 \pi \hbar c \varepsilon_{s}\left(Q^{2}\right)} .
$$

The subtracted dispersion relation for the strong color screening response function is governed by

$$
\begin{gathered}
\varepsilon_{s}\left(Q^{2}\right)=-\frac{Q^{2}}{\pi} \int_{0}^{\infty}\left[\frac{\mathfrak{I} m \varepsilon_{s}\left(-v-i 0^{+}\right)}{v+Q^{2}}\right] \frac{d v}{v}, \\
-\frac{\mathfrak{I} m \varepsilon_{s}\left(-v-i 0^{+}\right)}{\pi}=\frac{\alpha_{s}}{4 \pi}\left[\frac{11}{3} N_{c}-\frac{2}{3} n_{f}\right]+\ldots
\end{gathered}
$$


For QCD [24] it then follows that $\varepsilon_{s}\left(Q^{2}\right) \geq 0$ so there is no QCD Landau ghost. On the other hand, the color conductivity is not dissipative. In detail,

$$
\mathfrak{I} m \varepsilon_{s}\left(-\mathrm{v}-i 0^{+}\right) \leq 0 \quad \text { (color amplifier). }
$$

The perturbative QCD vacuum as described by Eqs.(15) and (16) is thereby unstable. The length scale $L$ associated with strong color screening $\varepsilon_{s}\left(Q^{2}\right)$, i.e.

$$
L^{2}=-2 \lim _{Q^{2} \rightarrow 0^{+}} \frac{\varepsilon_{s}\left(Q^{2}\right)}{Q^{2}} .
$$

The two body color potential (as $r \rightarrow \infty$ ) may then be written as a QCD string linear potential

$$
V_{s}(r)=-\eta^{a b} t_{1 a} t_{1 b} \sigma r \quad \text { where } \quad \sigma=\frac{g^{2}}{4 \pi L^{2}} .
$$

is the string tension.

\section{Nuclear Matter and String Models}

The liquid droplet model of a nucleus, assumes a non-relativistic dilute fluid of atomic mass $A$ and radius $R=a A^{1 / 3} \approx 1.2 \times 10^{-13} \mathrm{~cm} A^{1 / 3}$ filling up a Fermi sphere of momentum radius $p_{F}=\hbar k_{F}$,

$$
A=\left[\frac{4 \pi R^{3}}{3}\right]\left[\frac{4}{(2 \pi)^{2}}\right]\left[\frac{4 \pi k_{F}^{3}}{3}\right]=\frac{8}{9 \pi}\left(k_{F} R\right)^{3} .
$$

Eq.(19) allows for the computation of the Fermi velocity $v_{F}=\hbar k_{F} / M$; i.e.

$$
\frac{v_{F}}{c}=\left(\frac{9 \pi}{8}\right)^{1 / 3} \frac{\hbar}{M c a} \approx \frac{1}{4} .
$$

The liquid drop thereby appears to be internally relativistic. Another notion of nuclear liquids being relativistic arises in shell models. A strong $\mathbf{L} \cdot \mathbf{S}$ coupling exists characteristic of the Dirac relativistic spinor model of fermions. The QCD string constituents of nuclear matter starts from a relativistic viewpoint.

In the string model, the atomic number $Z=2 N_{u}+N_{d}$ and the atomic mass $A=3\left(N_{u}+N_{d}\right)$ can be expressed in terms of the net numbers of up and down quarks. The mesons internal to the nucleus are made of quark anti-quark pairs 
connected by strings. The strings consist of color electric flux tubes. The electric field colorless condensate $\mathcal{E}=\sqrt{\eta_{a b}\left\langle\mathbf{E}^{a} \cdot \mathbf{E}^{b}-\mathbf{B}^{a} \cdot \mathbf{B}^{b}\right\rangle}$ within the string determines the the magnitude of the string tension $\sigma=g \mathcal{E}$.

To understand the spectrum of excited states of a QCD string, one may consider a length $\Lambda$ of a rotating string along with an eigenvalue angular velocity

$$
\omega=\frac{\pi c}{\Lambda}=\frac{\pi c \sigma}{E}=\frac{\pi \sigma}{M c}
$$

wherein $E=M c^{2}=\sigma \Lambda$ is the energy of the string. Since the angular velocity of the string with angular momentum $J$ obeys

$$
\omega=\frac{d E}{d J}=\frac{\pi c \sigma}{E} \quad \Rightarrow \quad \frac{d J}{d\left(E^{2}\right)}=\frac{1}{2 \pi c \sigma},
$$

it follows that the angular momentum energy-squared variation is a linear classical Regge trajectory. The quantum mechanical version of Eq.(22) is usually written

$$
J=J_{0}+\left(\frac{E^{2}}{2 \pi c \sigma}\right)=\hbar\left(\alpha_{0}+\alpha^{\prime} M^{2}\right)=\hbar \alpha_{0}+\left(\frac{c^{3}}{2 \pi \sigma}\right) M^{2} .
$$

From the experimental slope of the meson spectrum, the measured value of the QCD string tension is given in Eq.(1). As the energy grows ever larger, the degeneracy of the excited states grows exponentially. The resulting increasing entropy and the large string energy degeneracy leads to notion of a Hagedorn temperature.

\section{String Entropy}

For a fixed angular momentum $J=\hbar N$ and for two polarizations

$$
N=\sum_{p=1,2} \sum_{k=1}^{\infty} k N_{k j}
$$

The number of partitions of the integer $N$ into the sum of other integers equal or less than $N$ yields the degeneracy number $\Omega[25,26]$. For large $N$,

$$
\begin{aligned}
\ln \Omega(N) & =2 \pi \sqrt{\frac{2 N}{3}}+\ln \left(\frac{3^{1 / 2}}{(6 N)^{7 / 4}}\right)+\ldots, \\
N & =\frac{E^{2}}{2 \pi \hbar c \sigma}+\ldots, \\
S(E) & =k_{B} \ln \Omega(N) .
\end{aligned}
$$




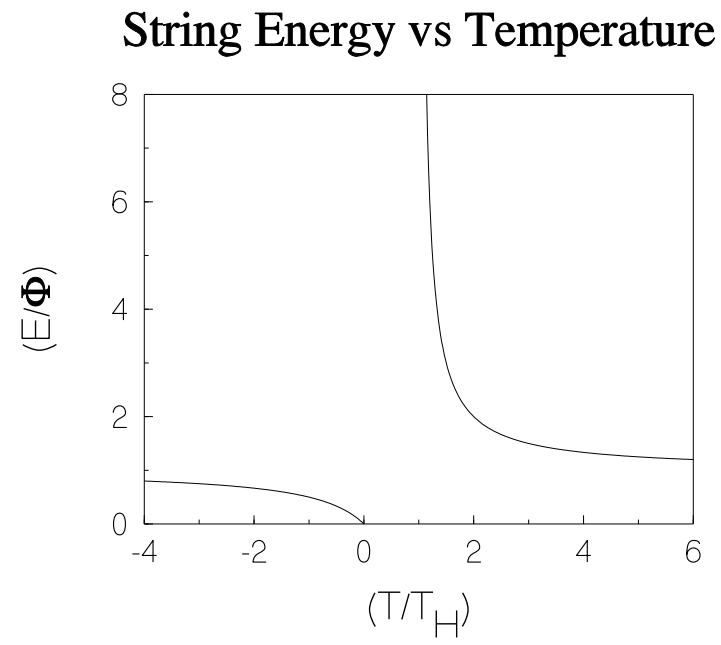

Figure 1: The temperature in the interval $0<T<T_{H}$ is forbidden. For a small amount of energy $E<\Phi$ fed into a string from a high energy nuclear collision, the temperature will be negative. In the high energy transfer regime, $E>\Phi$, the temperature will be positive. The temperature passes from negative to positive through the points $T= \pm \infty$ as $E$ passes through $\Phi$. For the very highest collision energy passed to a string, the temperature will "cool" down from above to $T_{H}$. The Hagedorn temperature is the lowest possible positive temperature of the string. In this regard, note the negative heat capacity $C=(d E / d T)<0$.

The microcanonical string entropy determines the temperature via

$$
\begin{array}{r}
\frac{1}{T}=\frac{d S}{d E} \text { and } k_{B} T_{H}=\sqrt{\frac{3 \hbar c \sigma}{4 \pi}}, \\
E=\left(\frac{T}{T-T_{H}}\right) \frac{7 k_{B} T_{H}}{2}=\left(\frac{T}{T-T_{H}}\right) \Phi .
\end{array}
$$

Eq.(26) describes the thermodynamic excitation energy above the Hagedorn temperature $k_{B} T_{H} \approx 207 \mathrm{MeV}$; i.e. $\lim _{T \rightarrow \pm \infty} E=\Phi \approx 725 \mathrm{MeV}$. The string energy is plotted as a function of temperature in Figure1

The following is a simple physical picture for describing how QCD strings behave during a high energy nuclear collisions. During a collision the meson (quark anti quark) or nucleon (three quark) string connections are excited. If a small amount of collision energy is transferred into a string, then the string temperature will be negative $0<E<\Phi \Rightarrow 0>T>-\infty$. If a large amount of collision 
energy is transferred into a string, then the string temperature will be positive $\Phi<E<\infty \Rightarrow T>T_{H}$ over and above the Hagedorn temperature. The string heat capacity

$$
C=\frac{d E}{d T}=-\frac{\Phi T_{H}}{\left(T-T_{H}\right)^{2}}<0 \quad(\mathrm{QCD} \text { string }) .
$$

The color glass-like time scales of string motions in the high energy limit are given in Eq.(5). As $T \rightarrow T_{H}+0^{+}$, the time scales grow ever larger consistent with the VFT law often applied to polymer and other glasses. The QCD strings in nuclear matter thereby play the role of polymer (or other network) chains in melted glass with viscosity

$$
\eta=\rho c^{2} \tau=\rho c^{2} \tilde{\tau} \exp \left[\frac{\Phi}{k_{B}\left(T-T_{H}\right)}\right], \quad\left(T>T_{H}\right),
$$

wherein $\rho$ is the fluid mass density. A color glass near the Hagedorn temperature is similar to a very viscous fluid. If the duration of a collision is sufficiently short, then there is little time to excite highly energetic string states leaving one with an intermediate temperature $T \gg T_{H}$ which implies a short time scale and a low viscosity. The resulting low viscosity in an almost ideal fluid is sometimes called a "perfect fluid"[27, 28].

Finally, we note in passing that a black hole has thermodynamic analogies to a QCD string; Black holes, as well as some observed normal stars, also have negative heat capacity; e.g. for the black hole

$$
\begin{array}{r}
\frac{S}{k_{B}}=4 \pi\left(\frac{G M^{2}}{\hbar c}\right)=4 \pi\left(\frac{G E^{2}}{\hbar c^{5}}\right), \\
\frac{d E}{d S}=T \Rightarrow E=\left(\frac{\hbar c^{5}}{8 \pi G}\right) \frac{1}{k_{B} T}=\left(\frac{\hbar c \sigma_{\text {gravity }}}{k_{B} T}\right), \\
C=\frac{d E}{d T}=-\left(\frac{\hbar c \sigma_{\text {gravity }}}{k_{B} T^{2}}\right)<0 .
\end{array}
$$

\section{String Fragmentation}

What happens to a highly excited state QCD string when it decays into final product particles in a nuclear collision? If the string is highly excited, then what appears in the final state products are jets of the many parts of the strings which 
fragment into lower mass elementary particles. To see what is involved, consider a meson made of a $q \bar{q}$ quark pair each with mass $m$ which sit on the ends of a QCD string. The quark pair Hamiltonian matrix may be written as

$$
H=\left(\begin{array}{cc}
c p & m c^{2} \\
m c^{2} & -c p
\end{array}\right) \text { with eigenvalues } E_{ \pm}(p)= \pm \sqrt{c^{2} p^{2}+m^{2} c^{4}}
$$

The transition rate per unit time to fragment a point on a string cereating a $q \bar{q}$ pair follows by applying Fermi's golden rule to the Hamiltonian matrix Eq.(30); i.e.

$$
\gamma=\left(\frac{2 \pi}{\hbar}\right)\left|m c^{2}\right|^{2} \delta(2 c p) .
$$

The probability of finding the fragmentation phase point $\in(d p d x) / 2 \pi \hbar$ is

$$
d^{2} P=\left(\frac{d p d x}{2 \pi \hbar}\right) e^{-\int \gamma d t} .
$$

Since the rate of change of momentum of a quark in a color condensate electric field is given by

$$
\frac{d p}{d t}=g \mathcal{E}=\sigma
$$

it follows that the transition per unit time per unit length for a QCD string to fragment producing a $q \bar{q}$ pair is given by

$$
\frac{d^{2} P}{d x d t}=\left(\frac{\sigma}{2 \pi \hbar}\right) \exp \left[-\frac{\pi m^{2} c^{3}}{\hbar \sigma}\right]
$$

which is exponentially suppressed if the quark mass $m$ is high.

\section{Conclusions}

It was shown how the perturbation theory QED vacuum is stable to decay but has a short space-like distance Landau ghost. The perturbation theory QCD vacuum has no Landau ghost, but is in an excited energetic state of negative temperature. From the viewpoint of QCD, nuclear matter consists of relativistic nucleons each with three quarks and relativistic mesons each with a quark anti quark pair. The quarks are tied together by gluons in the form of strings having tension $\sigma$. The excitation of QCD strings at low energy has a negative temperature and at high 
energy has a positive temperature always higher than $T_{H}$. Very high energy strings $T \rightarrow T_{H}+0^{+}$move very slowly as a viscous melted glass with very high viscosity. However, in a very short collision time it is difficult to transfer the initial collision kinetic energy into the internal energy of a few strings. The low energy strings at high $T \gg T_{H}$ have a low viscosity giving rise to an almost perfect fluid. The role of strings in a nucleus is closely analogous to the role of polymer chains in some viscous glass beads.

\section{References}

[1] L. Maiani, F. Piccinini, A.D. Polosa and V. Riquer, Nucl.Phys. A748, 209 (2005).

[2] E.V. Shuryak, Phys.Rev. D70, 054507 (2004).

[3] E.V. Shuryak, Nucl.Phys. A702, 83 (2002).

[4] S. Soff, S. A. Bass and A. Dumitru, Phys. Rev. Lett. 86, 3981 (2001).

[5] D. Teaney, J. Lauret, E.V. Shuryak, Phys.Rev.Lett. 86, 4783 (2001).

[6] M. Gyulassy and L. McLerran, Nucl. Phys. A750, 30 (2005).

[7] Dmitri Kharzeev and Kirill Tuchin, Nucl.Phys. A753, 316 (2005).

[8] E. Iancu and R. Venugopalan in QGP 3, R. Hwa and X.-N. Wang Editors, World Scientific, Singapore (2004).

[9] J. Jalilian-Marian, Mod. Phys. Lett. A19, 1251 (2004).

[10] Raju Venugopalan, hep-ph/0412396 (2004).

[11] E. Iancu, A. Leonidov, and L. McLerran, hep-ph/0202270 (2002).

[12] Raju Venugopalan, Comments Nucl. Part.Phys. 22, 113 (1997).

[13] A. Ayala, J. Jalilian-Marian, L. McLerran and R. Venugopalan, Phys. Rev. D52, 2935 (1995) .

[14] H. Vogel, Phys. Z. 22, 645 (1921). 
[15] G.S. Fulcher, J. Am. Chem. Soc. 8, 339 (1925); J. Am. Chem. Soc. 8, 789, (1925).

[16] G. Tammann and W. Hesse, Z. Anorg. Allg. Chem. 156, 245 (1926).

[17] H. Tanaka, Phys. Rev. Lett. 90, 055701 (2003).

[18] V. Perihar, A. Widom and Y.N. Srivastava, Phys. Rev. C73, 017901 (2006).

[19] R. Hagedorn, Nuovo Cim. Suppl. 3, 147 (1965).

[20] L.D. Landau, in Neils Bohr and the Development of Physics, W. Pauli Editor, Pergamon Press, London (1955).

[21] S. Sivasubramanian, Y. Srivastava and A. Widom, Mod. Phys. Lett. B16 1201 (2002).

[22] V.B. Berestetskii, E.M. Lifshitz and L.P. Pitaevskii, Quantum Electrodynamics, §114, Butterworth-Heinemann, Oxford (1997).

[23] Y. Srivastava and A. Widom, Phys. Rev. D63 077502 (2001).

[24] Y. Srivastava, S. Pacetti, G. Pancheri and A. Widom, "Dispersive techniques for $\alpha_{s}, R_{h a d}$ and instability of the perturbative vacuum", Invited talk at the conference " $e^{+} e^{-}$physics at intermediate energies workshop", eConf C010430 T19 (2001).

[25] G.H. Hardy and S. Ramanujan Proc. London Math. Soc. 17, 75 (1918)

[26] K. Huang and S. Weinberg, Phys. Rev. Lett. 25, 895 (1970).

[27] K. Adcox, et. el., Nucl. Phys. A757, 184 (2005).

[28] John Adams, et. al. ,Nucl. Phys. A757, 102 (2005). 